Wasser von 50" C., fïgt 3 cc Schwefelsäure $z u$, schiittelt eine halbe Minute, stellt wieder in die Wärme und liest nach einer halben Stunde ab, wonach die Grenze des Niederschlages sichtbar wird.

Nach $\mathrm{K}$ li m on müsste man, um annnähernd correct vorzugehen, für die Harzbestimmung mit Schwefelsäure im Ueberschuss versetzen, schütteln, in einem kleinen Scheidetrichter das Mineralöl abziehen, so scharf als dies möglich ist; den Rückstand verdiunt man mit Wasser, filtrirt in ein gewogenes Filter und bestimmt so das Harz.

Mischt man Oele von verschiedenem Harzgehalt, so zeigt sich, dass der Harzgehalt der Mischung grösseristals dasarithmetische Mittel der Harzgehalte der Componenten.

Gleiche Theile Nobel-Oel (2,5\% Harzg.) und Pale-Oil $(4 \%)$ zeigen $5 \%$ Harzehalt.

Gleiche Theile Nobel-Oel $(2,5 \%)$ und Red Engine-Oil $(7 \%$ ) zeigen $8,7 \%$ Harzgehalt.

Amerikanisches Spindel-Oel $(7 \% / 0)+$ Nobel-Oel $(2,5)$ zu gleichen Theilen: $7,5 \%$.

Galizischer Massut $\left(5^{8-}-56^{\circ} / 0\right)+$ Cylinderöl (o) zu gleichen Theilen: $32 \%$,

Liegen Gemische von Mineralölen mit vegetabilischen Oelen yor, so ist eine Harzgehaltsbestimmung unmöglich, da das vegetabilische Oel mit der Schwefelsäure reagirt.
So geben z. B. gleiche Theile Rüböl und Mineralöl $(8-9 \%)$ einen "Harzgehalt" von $88 \%$ !

In den cubicirten Cylindern setzt sich oft die Schwefelsäure nicht ganz so gut $a b$, wie in Gefässen von der Form eines Scheidetrichters. Empfehlenswerth ist für die Harzbestimmung der von $\mathrm{Cha}$ ritschkoff für die Bestimmung des Wassers in Rohnaphtha empfohlene einfache Apparat, der aus einer cubicirten Eprouvette (Theilung $10 \mathrm{cc}$ in $1 / 10 \mathrm{cc}$ ) und angeschmolzener Kugel mit Hals und Theilstrich I oo cc am Hals besteht. Setzt man in den Hals einen Kautschukstopfen mit Thermometer, so lässt sich gleichzeitig, ähnlich wie im Maumené-Apparat, die Wärmeentwicklung während der Reaction bestimmen.

$\mathrm{Ob}$ es praktisch ist, das Oel stärker als im Verhältniss $1:$ I mit Benzin zu verdünnen, oder bei diesem Verhältniss zu bleiben, darüber gehen die Meinungen sehr auseinander. Die Resultate stehen in keiner bestimmten Gesetzmässigkeit zur Verdünnung. Bei manchen Oelen wirkt die Verdünnung nicht auf den abgelesenen Theergehalt, bei anderen wieder drückt sie denselben herab. Acceptirt man daher tiberhaupt die Harzbestimmungsmethode, so ist es sehr dringend, eine einheitliche Vorschrift zur Ausfibrung des Versuches festzustellen.

(Schluss folgt.)

\title{
Ueber die Hübl'sche und Waller'sche Jodadditionsmethode.
}

\author{
Von Dr. Karl Dieterich, Helfenberg.
}

In dieser Zeitschrift ${ }^{1}$ ) hat unlängst R. Pelgr.y zu obiger Frage einen bemerkenswerthen Beitrag geliefert, der um so erwünschter erscheint, als die Wallersche Lösung thatsächlich verdient, der Hüblschen Lösung vorgezogen $z u$ werden; in diesem Sinn äussert sich ja auch R. Pelgry. Leider ist dem Verfasser ein kleiner Irrtum untergelaufen, der allerdings verzeihlich genug ist. Pelgry hat seine Daten aus den Helfenberger Annalen I 895 geschöpft und ist damit der Meinung, dass Karl Dieterich der Schreiber dieser Annalen gewesen ist. Die Helfenberger Annalen 1895 sind jedoch noch von Eugen Dieterich herausgegeben und die betreffenden von Pelgry citirten Stellen auf den Namen E. Dieterich zu setzen. Diese kleine Verwechslung thut selbstredend dem Werth der Pelgry'schen Arbeit keinen Abbruch; derselbe bestätigt ausserdem mit seinen Daten eine mit zahlreichem Zahlenmaterial belegte Arbeit von mir, die er selbst leider überseben hat. Ich sage leider, weil ich bereits vor

1) Chem. Revue $1897,78$. einem halben Jahre in dieser "Studie iiber die Huibl'sche Jodadditionsmethode und ihre Modification durch Waller" ${ }^{2}$ ), für die Waller'sche Lösung gesprochen und bereits - wenn auch auf anderen Wegen und auf Grund eines noch grösseren Zahlenmaterials - vor Pelgry zu ähnlichen Resultaten, wie dieser gelangt bin. Pelgry hat also meine Arbeit theilweise bestätigt, theilweise erweitert. Allerdings habe ich damals in Uebereinstimmung mit R. Henriques und Pelgry ausgeführt, dass noch grösseres Zahlenmaterial nöthig sei, um die Frage definitiv zu entscheiden.

Der Theorie nach müssten - so führte ich in meiner Studie aus - die Waller'schen Jodzahlen höher liegen, als die Hübl'schen Zahlen und zwar deshalb, weil der Salzsäure-Zusatz nach Waller selbst die theilweise Oxydation der ungesättigten Verbindungen verhindern soll, was einer grösseren Addition gleichbedeutend ist. Ich habe in Uebereinstimmung mit E. Dieterich gefunden, dass die Waller'schen

2) Pharm. Ztg. 1896, Nr. 93 . 
Jodzahlen theils niedriger, theils höher als die Hüblschen liegen, eine völlige Uebereinstimmung ist theoretisch auch nicht gut möglich und zwar aus obigem, von Waller selbst angeführtem Grunde. Immerhin haben unsere Versuche - dieselben sind seit 2 Jahren an über 1000 Oelen und Fetten im Gange - ergeben, dass die Abweichungen zwischen beiden Jodzahlen wohl vorhanden, aber doch nicht so erheblich sind, als dass man nicht die Wallersche Modification schon der grösseren Haltbarkeit wegen und aus den von mir in der erwähnten studie ausgefiihrten Gründen der Hübl'schen vorziehen dürfte. Die demnächst erscheinenden und von mir herausgegebenen Helfenberger Annalen i 896 entscheiden sich wiederum für die Waller'sche Modification. Die Arbeit von Pelgry und die Vorschläge von Henriques sind darum höchst willkommene Beiträge zur definitiven Entscheidung dieser Frage.

Ich fasse zum Schluss meine Ansichten über diesen Punkt kurz folgendermassen zusammen:

I. Die Waller'sche Iösung sollte theoretisch höhere Werthe erwarten lassen, als die Hübl'sche.

1I. Die Praxis hat ergeben, dass die W. Jodzahlen theils höher, theils niedriger liegen als die H. Jodzahlen.
III. Eine völlige Uebereinstimmung ist aus obigem theoretischen (irund (Waller'sche Auslegung) nicht zu erwarten, wenn auch die Praxis theilweise Uebereinstimmung zeigt.

IV. Die Studie von $\mathrm{K}$. Dieterich uiber diese Frage (Pharm. Ztg. No. 93) hat auch ergeben, dass die Waller'schen Jodzahlen der ,berechneten" Jodzahl in den iberwiegenden Fällen näher kommt, als die Hübl'schen Jodzahlen.

V. Die Waller'sche Jodlösung besitzt grössere Haltbarkeit als diejenige von Hübl.

Zieht man schliesslich noch die Resultate von Pelgry und Henriques hinzu - beide erhielten theilweise gut ibereinstimmende Resultate - so möchte man sich fast definitiv zu Gunsten der Waller'schen Modification entscheiden. Die schlechte Uebereinstimmung der Zahlen bei Harzöl ist mir übrigens deshalb nicht wunderbar, weil diese Harzöle sehr oft zur Erhöhung des spez. Gewichtes mit Colophonium verfälscht sind. Dass die Harze sehr unsichere Jodzahlen geben und dass man dann bei der Waller'schen und Hiibl'schen Lösung Differenzen von fast $100 \%$ erhält, habe ich an anderer Stelle (Ber. d. Pharm. Ges. I 896 , 140 und Pharm. Ztg. 1 $896 \mathrm{Nr} .93$ ) ausgefiihrt.

\section{BERICHTE.}

\section{Technologie.}

Das Gift der Baumwollsamen and Baumwollsamenkuchen von $\mathrm{Ch}$. Cornevin. Wäbrend der Verfasser die Ungiftigkeit des Baumwollsamenöls ebenfalls constatiren konnte, zeigt er, duss Thiere, beispielsweise junge Schweine, nach kurzdauernder Fütterung mit itgyptischen Baumıollsamen eingingen. Durch Uebergiessen der Samen mit Wasser konnte eine starkgiftige Flüssigkeit erbalten werden, dio bei subcutanel Eingabe kräftige Thiere schnell tödtete. Weitere Versuche zeigten, dass die Sebalen weniger schädlich waren, als clas reine, gelbe Mehl. (Ann. agron. 22,353 durch Chen. Centralbl. 1897, 516.).

inseres Wissens hat dio Fïtlerung mit Baumwollsimen-Presskuchen sich für manche Vichrassen sehr gut bowährt. Es ist allerdings scit jeher gobräuchlich, die Kuchen vor dem Gebrauch zu "wässern", wodurch, wie es scheint, giftige Extrartstoffe leicht $x u$ entferner sind.

Ueber Olivenkultureu. In Indien ist neuerdings eine Reihe interessanter Versuche abgeschlossen worden zum $Z$ weck, die Olive in den nördlichen Provinzen zu kultiviren, nachdem schon lange das Auftreten wildwachsender Qlivenbäune beobachtet worden war. Diese sind nun mit Augen der besten italienischen Pflanzen veredelt worden und haben so ein sehr gutes Resultat gegeben.

In Spanien werden Oliven rorzugsweise bei Cadix cultivirt. Man unterscheidet Oliven zum Essen und solche zur Oelproduction; erstere werden im September und Oktuber vor der Reife der Frucht geerntet und dann eingemacht. Es giebt davon zwei Sorten, Königs- und Manzanilla-Oliven, erstere sind gross und werden meist in den Vereinigten Stitaten verbraucht, die ManzanillaOliven werden nur in Spanien, Manzanilla und Cuba cultivirt. Die ölliefernden Oliven werden nicht vor Ende Norember gesammelt und gepresst. (British and colon. Drugg. 1897, No. 3 nach Apoth.-Ztg. 1897, 204.).

Die schottisehe Schieferöl-Industrie hat zur Verringerung der Productionskosten den Nebenproducten grössere Aufmerksamkeit, zugewendet, wozu der Gehalt mancher Schiefer an Anmoniak und Parraffin Anlass gab. Ihre Entwickelung in den letzten 30 Jahren zeigt folgende aus dem, Colliery Guardian" entnomıene Zusammonstellung.

\begin{tabular}{|c|c|c|c|}
\hline & 1871 & 1880 & 1890 \\
\hline Zahl dor Oelfabrike & 51 & 18 & 13 \\
\hline $\begin{array}{c}\text { Verarbeiteter Oel- } \\
\text { schiefer, Tons } \\
\text { Production: }\end{array}$ & 800000 & 850000 & 1869300 \\
\hline Rohöl Gallonen & 25000000 & 29000000 & 52876700 \\
\hline Leuchtöle & 11250000 & 11400000 & $21680 \cdot 000$ \\
\hline Schmieröle & 2500000 & 5000000 & 9000000 \\
\hline Paraffin, Tons & 5800 & 9200 & 22.846 \\
\hline Ammoniumsulfat, To & 2350 & 4750 & 18488 \\
\hline Angelegtes Kapital & $E$ & 1300000 & 2583982 \\
\hline $\begin{array}{l}\text { Bzscnarugte berg- } \\
\text { arbeiter }\end{array}$ & — & 2200 & 3500 \\
\hline
\end{tabular}

